Tôhoku Math. Journ.

33 (1981), 383-393.

\title{
A GENERAL INTERPOLATION THEOREM OF MARCINKIEWICZ TYPE
}

\author{
HIKOSABURo KoMATSU
}

(Received March 7, 1980)

The Marcinkiewicz interpolation theorem has been generalized, on the one hand, by Calderón [2] and Hunt [4] to quasi-linear operators from a couple of Lorentz spaces to another. After Lions and Peetre discussed interpolation of linear operators from a couple of Banach spaces to another, Krée [6] and Peetre-Sparr [7] have succeeded in generalizing the theory to (quasi-) linear operators from a couple of quasi-normed Abelian groups to another. On the other hand, the weak type assumptions at the end points of indices have also been generalized by Calderón [2] in the case of Lebesgue spaces and by De VoreRiemenschneider-Sharpley [3] in the case of normed spaces. We give here an interpolation theorem which generalizes all of the above results.

1. Real interpolation groups of a couple of quasi-normed Abelian groups. We recall some of the results of Peetre-Sparr [7] (see [1]).

Let $X$ be an Abelian group. A quasi-norm on $X$ is by definition a real-valued function \|\|$_{X}$ on $X$ satisfying the following conditions:

$$
\begin{gathered}
\|x\|_{X} \geqq 0, \text { and }\|x\|_{X}=0 \Leftrightarrow x=0 ; \\
\|-x\|_{X}=\|x\|_{X} ; \\
\|x+y\|_{X} \leqq \kappa\left(\|x\|_{X}+\|y\|_{X}\right),
\end{gathered}
$$

where $\kappa$ is a constant independent of $x$ and $y$. Such a quasi-norm is called a $\kappa$-quasi-norm. An Abelian group equipped with a quasi-norm is called a quasi-normed Abelian group.

If $(\Omega, \mathscr{L}, \mu)$ is a measure space, then for each $0<p \leqq \infty$ the Lebesgue space $L^{p}(\Omega)$ is a quasi-normed Abelian group under the $\kappa_{p}$ quasi-norm

$$
\|f\|_{L^{p}(\Omega)}= \begin{cases}\left(\int_{\Omega}|f(s)|^{p} d \mu(s)\right)^{1 / p}, & 0<p<\infty, \\ \underset{s \in \Omega}{\operatorname{ess} \sup }|f(s)|, & p=\infty,\end{cases}
$$

where 


$$
\kappa_{p}= \begin{cases}1, & 1 \leqq p \leqq \infty \\ 2^{(1-p) / p}, & 0<p<1 .\end{cases}
$$

When $(\Omega, \mathscr{C}, \mu)$ is the multiplicative group $(0, \infty)$ with the Haar measure $d s / s$, we write $L_{*}^{p}$ for $L^{p}(\Omega)$. In this case we also admit $\omega$ as an index and define $L_{*}^{\omega}$ to be the subspace of $L_{*}^{\infty}$ of all elements $f(s)$ such that $f(s) \rightarrow 0$ essentially as $s \rightarrow \infty$ and as $s \rightarrow 0$. The norm is the restriction of the norm of $L_{*}^{\infty}$. The index $\omega$ is defined to be greater than any finite $p$ but we do not define order relation between $\omega$ and $\infty$ to avoid confusion.

We define $\rho>0$ by $(2 \kappa)^{\rho}=2$. Then for each $\kappa$-quasi-norm \|\|$_{X}$ there is a 1-quasi-norm \|\|$_{x}^{*}$ such that

$$
\|x\|_{X}^{*} \leqq\|x\|_{X}^{o} \leqq 2\|x\|_{X}^{*} \text {. }
$$

Thus a natural uniform topology is introduced in the quasi-normed Abelian group $X$ by the metric $\|x-y\|_{X}^{*}$.

A pair of quasi-normed Abelian groups $\left(X_{0}, X_{1}\right)$ is said to be compatible if there is a Hausdorff topological group $\mathscr{X}$ for which continuous linear injections $i_{0}: X_{0} \rightarrow \mathscr{C}$ and $i_{1}: X_{1} \rightarrow \mathscr{C}$ are defined.

Let $X=\left(X_{0}, X_{1}\right)$ be a compatible couple of quasi-normed Abelian groups with $\kappa_{0}$-quasi-norm \|\|$_{X_{0}}$ and $\kappa_{1}$-quasi-norm \|\|$_{X_{1}}$. Then the sum $X_{0}+X_{1}$ in $\mathscr{X}$ is a quasi-normed Abelian group under

$$
\|x\|_{X_{0}+X_{1}}=\inf \left\{\left\|x_{0}\right\|_{X_{0}}+\left\|x_{1}\right\|_{X_{1}} ; x=x_{0}+x_{1}\right\} \text {, }
$$

which is a $\kappa$-quasi-norm with $\kappa=\max \left\{\kappa_{0}, \kappa_{1}\right\}$. We also define a $\kappa$-quasinorm $L(x, t)$ on $X_{0}+X_{1}$ with a parameter $0<t<\infty$ by

$$
L(x, t)=L_{X}(x, t)=\inf \left\{\left\|x_{0}\right\|_{X_{0}}+t^{-1}\left\|x_{1}\right\|_{X_{1}} ; x=x_{0}+x_{1}\right\} .
$$

This is nothing but $K\left(t^{-1}, x\right)$ of Peetre-Sparr [7] but more convenient in many respects. When an $x \in X_{0}+X_{1}$ is fixed, $L(x, t)$ is a positive, decreasing and continuous function of $t$.

If $0<\theta<1$ and $0<q \leqq \infty$ or $q=\omega$, the real interpolation group $X_{\theta, q}=\left(X_{0}, X_{1}\right)_{\theta, q}$ is defined to be the set of all $x \in X_{0}+X_{1}$ such that

$$
\|x\|_{X_{\theta, q}}=\left\|t^{\theta} L(x, t)\right\|_{L_{*}^{q}}<\infty \text {. }
$$

$X_{\theta, q}$ is a quasi-normed Abelian group under the quasi-norm $\|x\|_{X_{\theta, q}}$.

The index $q=\omega$ is often useful. For example, we have $\left(C^{0}, C^{1}\right)_{\theta, \infty}=$ $\operatorname{Lip}^{\theta}$ and $\left(C^{0}, C^{1}\right)_{\theta, \omega}=\operatorname{lip}^{\theta}$. For other examples see [5], where $\infty-$ is used instead of $\omega$.

If $0<q \leqq r$ or if $q=\omega$ and $r=\infty$, then we have the continuous inclusion $X_{\theta, q} \subset X_{\theta, r}$. This is an immediate consequence of the following lemma due to Hunt [4]. 
Lemma. Suppose that $f(t)$ is a non-negative and non-increasing function on $(0, \infty)$ and that $0<\theta<1$. If $t^{\theta} f(t)$ belongs to $L_{*}^{q}$, then it belongs to $L_{*}^{r}$ for any $r \geqq q$ and

$$
(\theta r)^{1 / r}\left\|t^{\theta} f(t)\right\|_{L_{*}^{r}} \leqq(\theta q)^{1 / q}\left\|t^{\theta} f(t)\right\|_{L_{*}^{q}} .
$$

If $(\Omega, \mathscr{C}, \mu)$ is a reasonable measure space, then the Lebesgue spaces $L^{p}(\Omega), p \geqq P$, are continuously imbedded in the Hausdorff topological vector space of all equivalence classes of measurable functions which belong to $L^{P}$ on each subset of finite measure. Thus $\left(L^{p_{0}}(\Omega)\right.$, $L^{p_{1}}(\Omega)$ ) is a compatible couple of quasi-normed Abelian groups for all $0<p_{i} \leqq \infty$.

For the couple $X=\left(L^{\infty}(\Omega), L^{p}(\Omega)\right)$ with $0<p<\infty$, Krée [6] and Bergh (see [1] p. 109) show that

$$
L_{X}(f, t) \sim\left(t^{-p} \int_{0}^{t^{p}}\left(f^{*}(s)\right)^{p} d s\right)^{1 / p},
$$

where $f^{*}(t)$ is the non-increasing rearrangement of $f(s)$. Hence we have the equivalence of interpolation groups $\left(L^{\infty}(\Omega), L^{p}(\Omega)\right)_{\theta, q}$ and Lorentz spaces $L^{(p / \theta, q)}(\Omega)$ for all $p \leqq q \leqq \infty$ or $q=\omega$. Here the Lorentz space $L^{(p, q)}(\Omega)$ is by definition the space of all equivalence classes of measurable functions $f(s)$ such that

$$
\|f\|_{L^{(p, q)(\Omega)}}=\left\|t^{1 / p} f^{*}(t)\right\|_{L_{*}^{q}}<\infty .
$$

In fact, suppose that $f \in L^{(p / \theta, q)}(\Omega)$ with $p \leqq q \leqq \infty$ or $q=\omega$. Then we have

$$
\begin{aligned}
\|f\|_{X_{\theta, q}} & =\left\|t^{\theta} L(f, t)\right\|_{L_{*}^{q}} \\
& \sim\left\|t^{\theta}\left[\int_{0}^{t^{p}}\left(s / t^{p}\right)\left(f^{*}(s)\right)^{p} d s / s\right]^{1 / p}\right\|_{L_{*}^{q}} \\
& =\left\|\left[\int_{0}^{t^{p}}\left(s / t^{p}\right)^{1-\theta}\left(s^{\theta / p} f^{*}(s)\right)^{p} d s / s\right]^{1 / p}\right\|_{L_{*}^{q}} \\
& =p^{-1 / q}\left\|\int_{0}^{u}(s / u)^{1-\theta}\left(s^{\theta / p} f^{*}(s)\right)^{p} d s / s\right\|_{L_{*}^{q / p}}^{1 / p} .
\end{aligned}
$$

Here we changed variable as $t^{p}=u$. Since the integral in the norm is the convolution on $(0, \infty)$ of the integrable function

$$
h(u)= \begin{cases}0, & 0<u<1, \\ u^{\theta-1}, & u \geqq 1,\end{cases}
$$

and $\left(s^{\theta / p} f^{*}(s)\right)^{p} \in L_{*}^{q / p}$, where $q / p \geqq 1$, the right hand side is bounded from above by 


$$
p^{-1 / q}(1-\theta)^{-1 / p}\left\|s^{\theta / p} f^{*}(s)\right\|_{L_{*}^{q}} .
$$

On the other hand, since $f^{*}(s)$ is non-increasing, the right hand side of (13) is bounded from below by

$$
p^{-1 / q}\left\|\left(f^{*}(u)\right)^{p} \int_{0}^{u}(s / u)^{1-\theta} s^{\theta} d s / s\right\|_{L_{*}^{q / p}}^{1 / p}=p^{-1 / q}\left\|u^{\theta / p} f^{*}(u)\right\|_{L_{*}^{q}} .
$$

Hence it follows that every $f \in\left(L^{\infty}(\Omega), L^{p}(\Omega)\right)_{\theta, q}$ belongs to $L^{(p / \theta, q)}(\Omega)$ and that two quasi-norms are equivalent.

2. The general interpolation theorem. We assume from now on that $X=\left(X_{0}, X_{1}\right)$ and $Y=\left(Y_{0}, Y_{1}\right)$ are compatible couples of quasi-normed Abelian groups and that $T$ is an operator defined on a subset $D(T)$ of $X_{0}+X_{1}$ and with values in $Y_{0}+Y_{1}$.

Definition 1. Let $\xi_{0}, \xi_{1}, \eta_{0}$ and $\eta_{1} \in[0,1]$ with $\xi_{0}<\xi_{1}$ and $\eta_{0} \neq \eta_{1}$ and let $r_{0}$ and $r_{1} \in(0, \infty)$. Then $T$ is said to be of generalized weak type $\left(\left(\xi_{0}, r_{0}\right), \eta_{0} ;\left(\xi_{1}, r_{1}\right), \eta_{1}\right)$ if there is a constant $M<\infty$ independent of $x \in D(T)$ such that

$$
\begin{aligned}
& L_{Y}(T x, t) \leqq M\left\{t^{-\eta_{0}}\left[\int_{t r}^{\infty}\left(s^{\varepsilon_{0}} L_{X}(x, s)\right)^{r_{0}} d s / s\right]^{1 / r_{0}}\right. \\
&\left.+t^{-\eta_{1}}\left[\int_{0}^{t r}\left(s^{\xi_{1}} L_{X}(x, s)\right)^{r_{1}} d s / s\right]^{1 / r_{1}}\right\},
\end{aligned}
$$

where

$$
\gamma=\left(\eta_{1}-\eta_{0}\right) /\left(\xi_{1}-\xi_{0}\right) .
$$

The generalized weak type $\left(p_{1}, q_{1} ; p_{2}, q_{2}\right)$ of De Vore-RiemenschneiderSharpley [3] is our generalized weak type $\left(\left(1 / p_{1}, 1\right), 1 / q_{1}\right) ;\left(\left(1 / p_{2}, 1\right), 1 / q_{2}\right)$.

We do not assume any kind of linearity of $T$. The main result of the present article is the following.

THEOREM 1. Suppose that $T$ is an operator of generalized weak type $\left(\left(\xi_{0}, r_{0}\right), \eta_{0} ;\left(\xi_{1}, r_{1}\right), \eta_{1}\right)$. Then for any $0<\theta<1$ and $0<q \leqq r \leqq \infty$ or $0<q \leqq r \leqq \omega$ there is a constant $C<\infty$ such that

$$
\|T x\|_{\eta_{\eta, r}} \leqq C\|x\|_{X_{\xi}, q}, \quad x \in D(T) \cap X_{\xi, q},
$$

where

$$
\xi=(1-\theta) \xi_{0}+\theta \xi_{1}, \quad \eta=(1-\theta) \eta_{0}+\theta \eta_{1} .
$$

Proof. Because of (10) it suffices to prove (16) only when $q=r$. First we consider the case where $q=r \geqq \max \left\{r_{0}, r_{1}\right\}$. We have by (14)

$$
\|T x\|_{Y_{\eta, q}} \leqq \kappa_{q} M\left\{\left\|t^{\eta-\eta_{0}}\left[\int_{t r}^{\infty}\left(s^{\hat{\varepsilon}_{0}} L(x, s)\right)^{r_{0}} d s / s\right]^{1 / r_{0}}\right\|_{L_{*}^{q}}\right.
$$




$$
\begin{gathered}
\left.+\left\|t^{\eta-\eta_{1}}\left[\int_{0}^{t \gamma}\left(s^{\xi_{1}} L(x, s)\right)^{r_{1}} d s / s\right]^{1 / r_{1}}\right\|_{L_{\star}^{q}}\right\} \\
=\kappa_{q}|\gamma|^{-1 / q} M\left\{\left\|\int_{u}^{\infty}(s / u)^{\left(\xi_{0}-\xi\right) r_{0}}\left(s^{\xi} L(x, s)\right)^{r_{0}} d s / s\right\|_{L_{*}^{q / r_{0}}}^{1 / r_{0}}\right. \\
\left.+\left\|\int_{0}^{u}(s / u)^{\left(\xi_{1}-\xi\right) r_{1}}\left(s^{\xi} L(x, s)\right)^{r_{1}}\right\|_{L_{\star}^{q / r_{1}}}^{1 / r_{1}}\right\} \\
\leqq \kappa_{q}|\gamma|^{-1 / q} M\left(\left(\left(\xi-\xi_{0}\right) r_{0}\right)^{-1 / r_{0}}+\left(\left(\xi_{1}-\xi\right) r_{1}\right)^{-1 / r_{1}}\right)\|x\|_{X_{\xi}, q} .
\end{gathered}
$$

The theorem in the general case is reduced to the above by the following.

Proposition 1. If $T$ is of generalized weak type $\left(\left(\xi_{0}, r_{0}\right), \eta_{0} ;\left(\xi_{1}, r_{1}\right)\right.$, $\left.\eta_{1}\right)$, then it is of generalized weak type $\left(\left(\xi_{0}, q_{0}\right), \eta_{0}\right.$; $\left.\left(\xi_{1}, q_{1}\right), \eta_{1}\right)$ for any $0<q_{0} \leqq r_{0}$ and $0<q_{1} \leqq r_{1}$.

Proof. Since $L(x, s)$ is decreasing in $s$, we have by Lemma

$$
\left[\int_{0}^{t r}\left(s^{\hat{1}_{1}} L(x, s)\right)^{r_{1}} d s / s\right]^{1 / r_{1}} \leqq\left(\xi_{1} r_{1}\right)^{-1 / r_{1}}\left(\xi_{1} q_{1}\right)^{1 / q_{1}}\left[\int_{0}^{t r}\left(s^{\xi_{1}} L(x, s)\right)^{q_{1}} d s / s\right]^{1 / q_{1}}
$$

Similarly we have

$$
\begin{aligned}
& {\left[\int_{t r}^{\infty}\left(s^{\xi_{0}} L(x, s)\right)^{r_{i}} d s / s\right]^{1 / r_{0}}} \\
& \quad \leqq\left(\xi_{0} r_{0}\right)^{-1 / r_{r}}\left(\xi_{0} q_{0}\right)^{1 / q_{0}}\left\{\int_{t r}^{\infty}\left(s^{\xi_{0}} L(x, s)\right)^{q_{0}} d s / s+\int_{0}^{t r}\left(s^{\xi_{0}} L\left(x, t^{r}\right)\right)^{q_{0}} d s / s\right\}^{1 / q_{0}} \\
& \quad \leqq \kappa_{q_{0}}\left(\xi_{0} r_{0}\right)^{-1 / r_{0}}\left(\xi_{0} q_{0}\right)^{1 / q_{0}}\left\{\left[\int_{t r}^{\infty}\left(s^{\xi_{0}} L(x, s)\right)^{q_{0}} d s / s\right]^{1 / q_{0}}+\left(\xi_{0} q_{0}\right)^{-1 / q_{0}} t^{r \xi_{0}} L\left(x, t^{r}\right)\right\} .
\end{aligned}
$$

For the second term we have

$$
t^{-\eta_{0}+{ }^{*} \hat{s}_{0}} L\left(x, t^{r}\right)=\left(\xi_{1} q_{1}\right)^{1 / q_{1}} t^{-\eta_{1}}\left[\int_{0}^{t r}\left(s^{\xi_{1}} L\left(x, t^{r}\right)\right)^{q_{1}} d s / s\right]^{1 / q_{1}} .
$$

Thus the right hand side of (14) is bounded by a constant times

$$
t^{-\eta_{0}}\left[\int_{t \gamma}^{\infty}\left(s^{\xi_{0}} L(x, s)\right)^{q_{0}} d s / s\right]^{1 / q_{0}}+t^{-\eta_{1}}\left[\int_{0}^{t \gamma}\left(s^{\xi_{1}} L(x, s)\right)^{q_{1}} d s / s\right]^{1 / q_{1}} .
$$

3. The Holmstedt theorem for quasi-linear operators. $T$ is assumed as above to be an operator from $D(T) \subset X_{0}+X$ into $Y_{0}+Y_{1}$.

Definition 2. $T$ is said to be quasi-linear if $x+y$ belongs to $D(T)$ whenever $x$ and $y$ belong to $D(T)$ and if there are constants $k$ and $c$ independent of $x$ and $y$ such that

$$
L_{Y}(T(x+y), t) \leqq k\left(L_{Y}(T x, c t)+L_{Y}(T y, c t)\right) .
$$

If $T$ is linear, then clearly (18) holds with $k=\kappa_{Y}$ and $c=1$. 
Krée [6] calls an operator $T$ with $D(T)=X_{0}+X_{1}$ quasi-linear if there are constants $k_{0}$ and $k_{1}$ such that for any $x_{0} \in X_{0}$ and $x_{1} \in X_{1}$ there are $y_{0} \in Y_{0}$ and $y_{1} \in Y_{1}$ satisfying

$$
T\left(x_{0}+x_{1}\right)=y_{0}+y_{1} \text { and }\left\|y_{i}\right\|_{Y_{i}} \leqq k_{i}\left\|x_{i}\right\|_{X_{i}} .
$$

This implies

$$
L_{Y}(T x, t) \leqq k L_{X}(x, t), \quad x \in X_{0}+X_{1},
$$

with $k=\max \left\{k_{0}, k_{1}\right\}$. Hence it follows that $T: X_{\theta, q} \rightarrow Y_{\theta, q}$ is bounded.

We consider, however, operators $T$ whose restrictions $T: X_{i} \rightarrow Y_{i}$ are not necessarily bounded.

Definition 3. Let $\xi, \eta \in[0,1]$ and $r \in(0, \infty] . \quad T$ is said to be of generalized weak type $((\xi, r), \eta)$ if there exists a constant $M<\infty$ such that

$$
\|T x\|_{Y \eta, \infty} \leqq M\|x\|_{X_{\xi, r}}, \quad x \in D(T) \cap X_{\xi, r} .
$$

If $\xi=0$ or 1 (resp. $\eta=0$ or 1 ), then we replace $X_{\xi, r}$ by $X_{\xi}$ (resp. $Y_{\eta, \infty}$ by $Y_{\eta}$ ).

If $T$ is of generalized weak type $((\xi, r), \eta)$, then it is clearly of generalized weak type $((\xi, q), \eta)$ for any $0<q \leqq r$.

The following theorem is due to Holmstedt [8] when $T$ is linear.

THEOREM 2. Let $\xi_{0}, \xi_{1}, \eta_{0}$ and $\eta_{1} \in[0,1]$ with $\xi_{0}<\xi_{1}$ and $\eta_{0} \neq \eta_{1}$ and let $r_{0}$ and $r_{1} \in(0, \infty)$. If a quasi-linear operator $T$ is simultaneously of generalized weak type $\left(\left(\xi_{0}, r_{0}\right), \eta_{0}\right)$ and $\left(\left(\xi_{1}, r_{1}\right), \eta_{1}\right)$, and if there is a constant a such that for every $x \in D(T)$ and $0<t<\infty$ there are $x_{0} \in$ $D(T) \cap X_{0}$ and $x_{1} \in D(T) \cap X_{1}$ satisfying $x=x_{0}+x_{1}$ and

$$
\left\|x_{0}\right\|_{X_{0}}+t^{-1}\left\|x_{1}\right\|_{X_{1}} \leqq a L_{X}(x, t),
$$

then $T$ is of generalized weak type $\left(\left(\xi_{0}, r_{0}\right), \eta_{0} ;\left(\xi_{1}, r_{1}\right), \eta_{1}\right)$ and, in particular, the conclusion of Theorem 1 holds.

PRoof. Let $x$ be an arbitrary element in $D(T)$. If we replace $a$ by a larger number, we can find a piecewise constant functions $x_{0}(t) \epsilon$ $D(T) \cap X_{0}$ and $x_{1}(t) \in D(T) \cap X_{1}$ such that

$$
\left\|x_{0}(t)\right\|_{X_{0}}+t^{-1}\left\|x_{1}(t)\right\|_{X_{1}} \leqq a L_{X}(x, t), \quad 0<t<\infty .
$$

Then applying (18) to $x=x_{0}\left(t^{r}\right)$ and $y=x_{1}\left(t^{r}\right)$, we have

$$
\begin{aligned}
L_{Y}(T x, t) & \leqq k L_{Y}\left(T x_{0}\left(t^{r}\right), c t\right)+k L_{Y}\left(T x_{1}\left(t^{r}\right), c t\right) \\
& \leqq k M_{0}(c t)^{-\eta_{0}}\left\|x_{0}\left(t^{r}\right)\right\|_{{\xi_{\xi_{0}}, r_{0}}_{0}}+k M_{1}(c t)^{-\eta_{1}}\left\|x_{1}\left(t^{r}\right)\right\|_{X_{\xi_{1}, r_{1}}} .
\end{aligned}
$$


The modifications necessary in the cases $\xi_{i}=0,1$ or $\eta_{i}=0,1$ would be obvious.

Now, in case $\xi_{0}>0$ we have

$$
\begin{aligned}
& t^{-\eta_{0}}\left\|x_{0}\left(t^{r}\right)\right\|_{X_{\xi_{0}}, r_{0}} \\
& \quad \leqq \kappa_{r_{0}} t^{-\eta_{0}}\left\{\left[\int_{t \gamma}^{\infty}\left(s^{\xi_{0}} L\left(x_{0}\left(t^{r}\right), s\right)\right)^{r_{0}} d s / s\right]^{1 / r_{0}}+\left[\int_{0}^{t r}\left(s^{\xi_{0}} L\left(x_{0}\left(t^{r}\right), s\right)\right)^{r_{0}} d s / s\right]^{1 / r_{0}}\right\} .
\end{aligned}
$$

Here we have

$$
\begin{aligned}
t^{-\eta_{0}}\left[\int_{t r}^{\infty}\left(s^{\xi_{0}} L\left(x_{0}\left(t^{r}\right), s\right)\right)^{r_{0}} d s / s\right]^{1 / r_{0}} \leqq \kappa_{X} t^{-\eta_{0}}\left[\int_{t r}^{\infty}\left(s^{\xi_{0}}\left(L(x, s)+L\left(x_{1}\left(t^{r}\right), s\right)\right)^{r_{0}} d s / s\right]^{1 / r_{0}}\right. \\
\quad \leqq \kappa_{X} \kappa_{r_{0}}\left\{t^{-\eta_{0}}\left[\int_{t \gamma}^{\infty}\left(s^{\xi_{0}} L(x, s)\right)^{r_{0}} d s / s\right]^{1 / r_{0}}+t^{-\eta_{0}}\left[\int_{t \gamma}^{\infty}\left(s^{\xi_{0}} L\left(x_{1}\left(t^{r}\right), s\right)\right)^{r_{0}} d s / s\right]^{1 / r_{0}}\right\} .
\end{aligned}
$$

Since $L\left(x_{1}\left(t^{r}\right), s\right) \leqq s^{-1}\left\|x_{1}\left(t^{r}\right)\right\|_{X_{1}}$,

$$
\begin{aligned}
& t^{-\eta_{0}}\left[\int_{t r}^{\infty}\left(s^{\xi_{0}} L\left(x_{1}\left(t^{r}\right), s\right)\right)^{r_{0}} d s / s\right]^{1 / r_{0}} \\
& \leqq\left(\left(1-\xi_{0}\right) r_{0}\right)^{-1 / r_{0}} t^{-\eta_{0}+r\left(\xi_{0}-1\right)}\left\|x_{1}\left(t^{r}\right)\right\|_{X_{1}} \\
& \leqq a\left(\left(1-\xi_{0}\right) r_{0}\right)^{-1 / r_{0}} t^{-\eta_{1}+r \xi_{1}} L\left(x, t^{r}\right) \\
& \leqq a\left(\left(1-\xi_{0}\right) r_{0}\right)^{-1 / r_{0}}\left(\xi_{1} r_{1}\right)^{1 / r_{1}} t^{-\eta_{1}}\left[\int_{0}^{t r}\left(s^{\xi_{1}} L(x, s)\right)^{r_{1}} d s / s\right]^{1 / r_{1}} .
\end{aligned}
$$

Here we employed the fact that $L(x, s)$ is decreasing.

Similarly we have

$$
\begin{aligned}
t^{-\eta_{0}}\left[\int _ { 0 } ^ { t r } \left(s^{\xi_{0}}\right.\right. & \left.\left.L\left(x_{0}\left(t^{\gamma}\right), s\right)\right)^{r_{0}} d s / s\right]^{1 / r_{0}} \\
& \leqq t^{-\eta_{0}}\left[\int_{0}^{t r}\left(s^{\xi_{0}}\left\|x_{0}\left(t^{\gamma}\right)\right\|_{X_{0}}\right)^{r_{0}} d s / s\right]^{1 / r_{0}} \\
& \leqq a\left(\xi_{0} r_{0}\right)^{-1 / r_{0}} t^{-\eta_{0}+\xi_{0}} L\left(x, t^{\gamma}\right) \\
& \leqq a\left(\xi_{0} r_{0}\right)^{-1 / r_{0}}\left(\xi_{1} r_{1}\right)^{1 / r_{1}} t^{-\eta_{1}}\left[\int_{0}^{t \gamma}\left(s^{\xi_{1}} L(x, s)\right)^{r_{1}} d s / s\right]^{1 / r_{1}} .
\end{aligned}
$$

In case $\xi_{0}=0$ we have

$$
\begin{aligned}
t^{-\eta_{0}}\left\|x_{0}\left(t^{r}\right)\right\|_{X_{0}} & \leqq a t^{-\eta_{0}} L\left(x, t^{\gamma}\right) \\
& \leqq a\left(\xi_{1} r_{1}\right)^{1 / r_{1}} t^{-\eta_{1}}\left[\int_{0}^{t r}\left(s^{\xi_{1}} L(x, s)\right)^{r_{1}} d s / s\right]^{1 / r_{1}} .
\end{aligned}
$$

Thus the first term of the right hand side of (24) is bounded by a constant multiple of the right hand side of (14).

The second term of $(24)$ is estimated similarly. We employ the inequality 


$$
t^{\gamma \varepsilon_{0}} L\left(x, t^{r}\right) \leqq\left(\left(1-\xi_{0}\right) r_{0}\right)^{1 / r_{0}}\left[\int_{t r}^{\infty}\left(s^{\varepsilon_{0}} L(x, s)\right)^{r_{0}} d s / s\right]^{1 / r_{0}},
$$

which is obtained from the fact that $s L(x, s)$ is increasing.

4. Applications. First we prove the reiteration theorem of PeetreSparr [7] as an application of Theorem 2.

TheOREM 3. Suppose that $X=\left(X_{0}, X_{1}\right)$ and $Y=\left(Y_{0}, Y_{1}\right)$ are compatible couples of quasi-normed Abelian groups and that $0 \leqq \theta_{0}<\theta_{1} \leqq 1$. Let $0<\eta<1$ and $0<q \leqq \infty$ or $q=\omega$ be arbitrary numbers and let

$$
\theta=(1-\eta) \theta_{0}+\eta \theta_{1} \text {. }
$$

(1) If $Y_{i} \subset X_{\theta_{i}, \infty}, i=0,1$, then

$$
Y_{\eta, q} \subset X_{\theta, q}
$$

(2) If $X_{\theta_{i}, q_{i}} \subset Y_{i}, i=0,1$, for some $0<q_{i} \leqq \omega$ or $\infty$, then

$$
X_{\theta, q} \subset Y_{\eta, q}
$$

(3) If $X_{\theta_{i}, q_{i}} \subset Y_{i} \subset X_{\theta_{i}, \infty}, i=0,1$, for some $0<q_{i} \leqq \omega$ or $\infty$, then

$$
Y_{\eta, q}=X_{\theta, q} \text {. }
$$

Here the inclusion $A \subset B$ means that the quasi-normed Abelian group $A$ is included in the quasi-normed Abelian group $B$ and there exists a constant $M$ such that

$$
\|a\|_{B} \leqq M\|a\|_{A}, \quad a \in A,
$$

and $A=B$ means that $A$ and $B$ are the same Abelian group with equivalent quasi-norms.

If $\theta_{0}=0$ (resp. $\theta_{1}=1$ ), then $X_{\theta_{0}, \infty}$ and $X_{\theta_{0}, q_{0}}$ (resp. $X_{\theta_{1}, \infty}$ and $X_{\theta_{1}, q_{1}}$ ) should be replaced by $X_{0}$ (resp. $X_{1}$ ).

Proof. (1) Define the operator $T: Y_{0}+Y_{1} \rightarrow X_{0}+X_{1}$ by

$$
T\left(y_{0}+y_{1}\right)=y_{0}+y_{1}, \quad y_{i} \in Y_{i} \text {. }
$$

This is a linear injective operator of generalized weak types $\left((0, *), \theta_{0}\right)$ and $\left((1, *), \theta_{1}\right)$ simultaneously. Hence it follows from Theorem 2 that the identity operator $T: Y_{\eta, q} \rightarrow X_{\theta, q}$ is bounded.

(2) In this case the identity operator $T: X_{\theta_{0}, q_{0}}+X_{\theta_{1}, q} \rightarrow Y_{0}+Y_{1}$ is linear and simultaneously of generalized weak type $\left(\left(\theta_{0}, q_{0}\right), 0\right)$ and $\left(\left(\theta_{1}, q_{1}\right), 1\right)$. Hence $T: X_{\theta, q} \rightarrow Y_{\eta, q}$ is bounded.

Let $(\Omega, \mathscr{K}, \mu)$ be a measure space. As we have shown in $\S 1$, 


$$
\left(L^{\infty}(\Omega), L^{p}(\Omega)\right)_{\theta, r}=L^{(p / \theta, r)}(\Omega)
$$

for any $r \geqq p$. Since $p$ can be chosen arbitrarily small, the reiteration theorem verifies the following.

Proposition 2. Let $0<p_{1}<p_{0} \leqq \infty$ and $q_{0}, q_{1} \in(0, \infty] \cup\{\omega\}$. Then for any $0<\theta<1$ and $0<r \leqq \infty$ or $r=\omega$ we have

$$
\left(L^{\left(p_{0}, q_{0}\right)}(\Omega), L^{\left(p_{1}, q_{1}\right)}(\Omega)\right)_{\theta, r}=L^{(p, r)}(\Omega),
$$

where

$$
\frac{1}{p}=\frac{1-\theta}{p_{0}}+\frac{\theta}{p_{1}}
$$

Lastly we show that the interpolation theorem of Calderón [2] and Hunt [4] is a consequence of Theorem 2.

Definition 4 . Let $(\Omega, \mathscr{L}, \mu)$ and $\left(\Omega^{\prime}, \mathscr{L}^{\prime}, \mu^{\prime}\right)$ be two measure spaces and let $T$ be an operator with the domain $D(T)$ in the space of (equivalence classes of) measurable functions on $\Omega$ and the range in the space of (equivalence classes of) measurable functions on $\Omega^{\prime}$. $T$ is said to be quasi-linear if $f+g \in D(T)$ whenever $f$ and $g \in D(T)$ and if there exists a constant $K$ independent of $f$ and $g$ such that

$$
|T(f+g)| \leqq K(|T f|+|T g|) \text {, a.e. }
$$

THEOREM 4. Let $T$ be a quasi-linear operator from the domain $D(T)$ of measurable functions on $\Omega$ into the space of measurable functions on $\Omega^{\prime}$ and let $p_{0}, p_{1}, q_{0}, q_{1} \in(0, \infty]$ with $p_{1}<p_{0}$ and $q_{0} \neq q_{1}$. If for each $f(s) \in D(T)$ and $m>0$ the truncations

$$
\begin{aligned}
& f_{0}(s)= \begin{cases}f(s), & |f(s)| \leqq m, \\
\frac{f(s)}{|f(s)|} m, & |f(s)|>m,\end{cases} \\
& f_{1}(s)= \begin{cases}0, & |f(s)| \leqq m, \\
f(s)-\frac{f(s)}{|f(s)|} m, & |f(s)|>m,\end{cases}
\end{aligned}
$$

belong to $D(T)$ and if there are constants $M_{0}, M_{1}, r_{0}, r_{1}>0$ such that

$$
\begin{aligned}
& \|T f\|_{L^{\left(q_{0}, \infty\right)}\left(\Omega^{\prime}\right)} \leqq M_{0}\|f\|_{L^{\left(p_{0}, r_{0}\right)}(\Omega)}, \\
& \|T f\|_{L^{\left(q_{1}, \infty\right)}\left(\Omega^{\prime}\right)} \leqq M_{1}\|f\|_{L^{\left(p_{1}, r_{1}\right)}(\Omega)}
\end{aligned}
$$

for all $f \in D(T)$, then for every $0<\theta<1$ and $0<r \leqq \infty$ or $r=\omega$ there is a constant $M$ such that 


$$
\|T f\|_{L^{(q, r)}\left(\Omega^{\prime}\right)} \leqq M\|f\|_{L^{(p, r)}(\Omega)}
$$

for all $f \in D(T)$, where

$$
\frac{1}{p}=\frac{1-\theta}{p_{0}}+\frac{\theta}{p_{1}}, \quad \frac{1}{q}=\frac{1-\theta}{q_{0}}+\frac{\theta}{q_{1}} .
$$

Proof. Let $0<P<\min \left\{p_{0}, p_{1}\right\}$ and $0<Q<\min \left\{q_{0}, q_{1}\right\}$ and regard $T$ as an operator from the couple $X=\left(L^{\infty}(\Omega), L^{P}(\Omega)\right)$ into the couple $Y=\left(L^{\infty}\left(\Omega^{\prime}\right), L^{Q}\left(\Omega^{\prime}\right)\right)$.

The quasi-linearity condition (31) implies

$$
(T(f+g))^{*}(t) \leqq K\left\{(T f)^{*}(t / 2)+(T g)^{*}(t / 2)\right\} .
$$

Since $L_{Y}(h, t) \sim\left[t^{-Q} \int_{0}^{t^{Q}}\left(h^{*}(s)\right)^{Q} d s\right]^{1 / Q}$, it follows that $T$ is quasi-linear in the sense of Definition 2.

In view of Proposition 2, conditions (34) and (35) say that $T$ is simultaneously of generalized weak type $\left(\left(P / p_{0}, r_{0}\right), Q / q_{0}\right)$ and $\left(\left(P / p_{1}, r_{1}\right)\right.$, $\left.Q / q_{1}\right)$.

Lastly, since the infimum $L_{X}(f, t)=\inf \left\{\left\|f_{0}\right\|_{L^{\infty}(\Omega)}+t^{-1}\left\|f_{1}\right\|_{L^{P}(\Omega)} ; f=\right.$ $\left.f_{0}+f_{1}\right\}$ is attained by some truncations (32) and (33) for each $t$, every $f \in D(T) \cap\left(L^{\infty}(\Omega)+L^{P}(\Omega)\right)$ has a decomposition $f=f_{0}+f_{1}$ with $f_{0} \in$ $D(T) \cap L^{\infty}(\Omega)$ and $f_{1} \in D(T) \cap L^{P}(\Omega)$ such that

$$
\left\|f_{0}\right\|_{L^{\infty}(\Omega)}+t^{-1}\left\|f_{1}\right\|_{L^{P}(\Omega)}=L_{X}(f, t) .
$$

Hence it follows from Theorems 1 and 2 that there exists a constant $C<\infty$ such that

$$
\|T f\|_{Y_{Q^{\prime}, r}} \leqq C\|f\|_{X_{P / p, r}}, \quad f \in D(T) \cap X_{P / p, r} .
$$

Since $X_{P / p, r}=L^{(p, r)}(\Omega)$ and $Y_{Q / q, r}=L^{(q, r)}\left(\Omega^{\prime}\right)$ by Proposition 2, we have (36).

\section{REFERENCES}

[1] J. Bergh AND J. Löfström, Interpolation Spaces, An Introduction, Springer-Verlag, Berlin-Heidelberg-New York, 1976.

[2] A. P. CALDERÓN, Spaces between $L^{1}$ and $L^{\infty}$ and the theorem of Marcinkiewicz, Studia Math. 26 (1966), 273-299.

[3] R. A. De Vore, S. D. Riemenschneider and R. C. Sharpley, Weak interpolation in Banach spaces, J. Functional Analysis 33 (1979), 58-94.

[4] R. A. Hunt, On $L(p, q)$ spaces, Enseignement Math. 12 (1966), 249-276.

[5] H. Komatsu, Fractional powers of operators II, Interpolation spaces, Pacific J. Math. 21 (1967), 89-111.

[6] P. KRÉE, Interpolation d'espaces qui ne sont ni normés, ni complets, Applications, Ann. Inst. Fourier 17 Fasc. 2 (1967), 137-174.

[7] J. Peetre ANd G. Sparr, Interpolation of normed Abelian groups, Ann. Mat. Pura 
Appl. 92 (1972), 217-262.

[8] T. HolmSTEDT, Interpolation of quasi-normed spaces, Math. Scand. 26 (1970), 177-199.

Department of Mathematics

FACULTY OF SCIENCE

UNIVERSITY OF TOKYO

HoNGo, TOKYo 113

JAPAN 
